\title{
The Crossing Number of Seq-Shellable Drawings of Complete Graphs
}

\author{
Petra Mutzel and Lutz Oettershagen \\ Department of Computer Science \\ TU Dortmund University
}

\begin{abstract}
The Harary-Hill conjecture states that for every $n>0$ the complete graph on $n$ vertices $K_{n}$, the minimum number of crossings over all its possible drawings equals

$$
H(n):=\frac{1}{4}\left\lfloor\frac{n}{2}\right\rfloor\left\lfloor\frac{n-1}{2}\right\rfloor\left\lfloor\frac{n-2}{2}\right\rfloor\left\lfloor\frac{n-3}{2}\right\rfloor .
$$

So far, the lower bound of the conjecture could only be verified for arbitrary drawings of $K_{n}$ with $n \leq 12$. In recent years, progress has been made in verifying the conjecture for certain classes of drawings, for example 2-page-book, $x$-monotone, $x$-bounded, shellable and bishellable drawings. Up to now, the class of bishellable drawings was the broadest class for which the Harary-Hill conjecture has been verified, as it contains all beforehand mentioned classes. In this work, we introduce the class of seq-shellable drawings and verify the Harary-Hill conjecture for this new class. We show that bishellability implies seq-shellability and exhibit a non-bishellable but seq-shellable drawing of $K_{11}$, therefore the class of seq-shellable drawings strictly contains the class of bishellable drawings.
\end{abstract}

\section{Introduction}

Let $G=(V, E)$ be an undirected graph and $K_{n}$ the complete graph on $n>0$ vertices. The crossing number $\operatorname{cr}(G)$ of $G$ is the smallest number of edge crossings over all possible drawings of $G$. In a drawing $D$ every vertex $v \in V$ is represented by a point and every edge $u v \in E$ with $u, v \in V$ is represented by a simple curve connecting the corresponding points of $u$ and $v$. The Harary-Hill conjecture states the following.

Conjecture 1 (Harary-Hill [8]). Let $K_{n}$ be the complete graph with $n$ vertices, then

$$
\operatorname{cr}\left(K_{n}\right)=H(n):=\frac{1}{4}\left\lfloor\frac{n}{2}\right\rfloor\left\lfloor\frac{n-1}{2}\right\rfloor\left\lfloor\frac{n-2}{2}\right\rfloor\left\lfloor\frac{n-3}{2}\right\rfloor .
$$

There are construction methods for drawings of $K_{n}$ that lead to exactly $H(n)$ crossings, for example the class of cylindrical drawings first described by Harary and Hill [9]. For a cylindrical drawing, we put $\left\lfloor\frac{n}{2}\right\rfloor$ vertices on the top rim and the remaining $\left\lceil\frac{n}{2}\right\rceil$ vertices on the bottom rim of a cylinder. Edges between 
vertices on the same rim (lid or bottom) are connected with straight lines on the lid or bottom. Two vertices on opposite rims are connected with an edge along the geodesic between the two vertices. The drawing of $K_{6}$ in figure 1 (a) is homeomorphic to a planarized cylindrical drawing of $K_{6}$.

However, there is no proof for the lower bound of the conjecture for arbitrary drawings of $K_{n}$ with $n>12$. The cases for $n \leq 10$ are shown by Guy [8] and for $n=11$ by Pan and Richter [12]. Guy [8] argues that $\operatorname{cr}\left(K_{2 n+1}\right) \geq$ $H(2 n+1)$ implies $\operatorname{cr}\left(K_{2(n+1)}\right) \geq H(2(n+1))$, hence $c r\left(K_{12}\right) \geq H(12)$. McQuillan et al. showed that $\operatorname{cr}\left(K_{13}\right) \geq 219$ [11]. Ábrego et al. 44 improved the result to $\operatorname{cr}\left(K_{13}\right) \in\{223,225\}$.

Beside these results for arbitrary drawings, there has been success in proving the Harary-Hill conjecture for different classes of drawings. So far, the conjecture has been verified for 2-page-book [1] $x$-monotone [26]15], $x$-bounded [2], shellable [2] and bishellable drawings [5]. The class of bishellable drawings comprises all beforehand mentioned classes, and until now it was the largest class of drawings for which the Harary-Hill conjecture has been verified. Ábrego et al. [5] showed that the Harary-Hill conjecture holds for bishellable drawings using cumulated $k$-edges.

Our contribution. In this work, we introduce the new class of seq-shellable drawings and verify the Harary-Hill conjecture for this new class. We show that bishellability implies seq-shellability and exhibit a drawing of $K_{11}$ which is seq-shellable but not bishellable. Therefore, we establish that the class of seqshellable drawings is strictly larger than the class of bishellable drawings.

The outline of this paper is as follows. In section 2 we present the preliminaries, and in particular the background on $k$-edges, cumulated $k$-edges and their usage for verifying the Harary-Hill conjecture. In section 3 we define simple sequences and their usage for proving lower bounds on the number of invariant edges. We present the definition of seq-shellability, verify the Harary-Hill conjecture for the new class and show its superiority towards the class of bishellable drawings. Finally, in section 4 we draw our conclusion and close with open questions.

\section{Preliminaries}

Formally, a drawing $D$ of a graph $G$ on the plane is an injection $\phi$ from the vertex set $V$ into the plane, and a mapping of the edge set $E$ into the set of simple curves, such that the curve corresponding to the edge $e=u v$ has endpoints $\phi(u)$ and $\phi(v)$, and contains no other vertices [14. We call an intersection point of the interior of two edges a crossing and a shared endpoint of two adjacent edges is not considered a crossing. The crossing number $\operatorname{cr}(D)$ of a drawing $D$ equals the number of crossings in $D$ and the crossing number $\operatorname{cr}(G)$ of a graph $G$ is the minimum crossing number over all its possible drawings. We restrict our discussions to good drawings of $K_{n}$, and call a drawing good if (1) any two of the curves have finitely many points in common, (2) no two curves have a 
point in common in a tangential way, (3) no three curves cross each other in the same point, (4) any two edges cross at most once and (5) no two adjacent edges cross. It is known that every drawing with a minimum number of crossings is good [13. In the discussion of a drawing $D$, we call the points also vertices, the curves edges and $V$ denotes the set of vertices (i.e. points), and $E$ denotes the edges (i.e curves) of $D$. If we subtract the drawing $D$ from the plane, a set of open discs remain. We call $\mathcal{F}(D):=\mathbb{R}^{2} \backslash D$ the set of faces of the drawing $D$. If we remove a vertex $v$ and all its incident edges from $D$, we get the subdrawing $D-v$. Moreover, we might consider the drawing to be on the surface of the sphere $S^{2}$, which is equivalent to the drawing on the plane due to the homeomorphism between the plane and the sphere minus one point.

In [5] Ábrego et al. introduce bishellable drawings.

Definition 1 (Bishellability [5]). For a non-negative integer $s$, a drawing $D$ of $K_{n}$ is s-bishellable if there exist sequences $a_{0}, a_{1}, \ldots, a_{s}$ and $b_{s}, b_{s-1}, \ldots$, $b_{1}, b_{0}$, each sequence consisting of distinct vertices of $K_{n}$, so that with respect to a reference face $F$ :

(i) For each $i \in\{0, \ldots, s\}$, the vertex $a_{i}$ is incident to the face of $D-\left\{a_{0}, a_{1}, \ldots, a_{i-1}\right\}$ that contains $F$,

(ii) for each $i \in\{0, \ldots, s\}$, the vertex $b_{i}$ is incident to the face of $D-\left\{b_{0}, b_{1}, \ldots, b_{i-1}\right\}$ that contains $F$, and

(iii) for each $i \in\{0, \ldots, s\}$, the set $\left\{a_{0}, a_{1}, \ldots a_{i}\right\} \cap\left\{b_{s-i}, b_{s-i-1}, \ldots, b_{0}\right\}$ is empty.

The class of bishellable drawings contains all drawings that are $\left(\left\lfloor\frac{n}{2}\right\rfloor-2\right)$ bishellable. In order to show that if a drawing $D$ is $\left(\left\lfloor\frac{n}{2}\right\rfloor-2\right)$-bishellable, the Harary-Hill conjecture holds for $D$, Ábrego et al. use the notion of $k$-edges. The origins of $k$-edges lie in computational geometry and problems over $n$-point set, especially problems on halving lines and $k$-set [3]. An early definition in the geometric setting goes back to Erdős et al [7. Given a set $P$ of $n$ points in general position in the plane, the authors add a directed edge $e=\left(p_{i}, p_{j}\right)$ between the two distinct points $p_{i}$ and $p_{j}$, and consider the continuation as line that separates the plane into the left and right half plane. There is a (possibly empty) point set $P_{L} \subseteq P$ on the left side of $e$, i.e. left half plane. Erdôs et al. assign $k:=\min \left(\left|P_{L}\right|,\left|P \backslash P_{L}\right|\right)$ to $e$. Later, the name $k$-edge emerged and Lovász et al. [10] used $k$-edges for determining a lower bound on the crossing number of rectilinear graph drawings. Finally, Ábrego et al. [1] extended the concept of $k$-edges from rectilinear to topological graph drawings.

Every edge in a good drawing $D$ of $K_{n}$ is a $k$-edge with $k \in\left\{0, \ldots,\left\lfloor\frac{n}{2}\right\rfloor-1\right\}$. Let $D$ be on the surface of the sphere $S^{2}$, and $e=u v$ be an edge in $D$ and $F \in \mathcal{F}(D)$ be an arbitrary but fixed face; we call $F$ the reference face. Together with any vertex $w \in V \backslash\{u, v\}$, the edge $e$ forms a triangle $u v w$ and hence a closed curve that separates the surface of the sphere into two parts. For an arbitrary but fixed orientation of $e$ one can distinguish between the left part and the right part of the separated surface. If $F$ lies in the left part of the surface, we say the triangle has orientation + else it has orientation - . For $e$ there are $n-2$ 
possible triangles in total, of which $0 \leq i \leq n-2$ triangles have orientation + (or - ) and $n-2-i$ triangles have orientation - (or + respectively). We define $k:=\min (i, n-2-i)$ and say $e$ is an $k$-edge with respect to the reference face $F$ and its $k$-value equals $k$ with respect to $F$. Ábrego et al. [1 show that the crossing number of a drawing is expressible in terms of the number of $k$-edges for $0 \leq k \leq\left\lfloor\frac{n}{2}\right\rfloor-1$ with respect to the reference face. The following definition of the cumulated number of $k$-edges is helpful in determining the lower bound of the crossing number.

Definition 2 (Cumulated $k$-edges [1]). Let $D$ be good drawing and $E_{k}(D)$ be the number of $k$-edges in $D$ with respect to a reference face $F \in \mathcal{F}(D)$ and for $k \in\left\{0, \ldots,\left\lfloor\frac{n}{2}\right\rfloor-1\right\}$. We call

$$
E_{\leq \leq k}(D):=\sum_{i=0}^{k}(k+1-i) E_{i}(D)
$$

the cumulated number of $k$-edges with respect to $F$.

We also write cumulated $k$-edges or cumulated $k$-value instead of cumulated number of $k$-edges. Lower bounds on $E_{\leq \leq k}(D)$ for $0 \leq k \leq\left\lfloor\frac{n}{2}\right\rfloor-2$ translate directly into a lower bound for $\operatorname{cr}(D)$.

Lemma 1. 1. Let $D$ be a good drawing of $K_{n}$ and $F \in \mathcal{F}(D)$. If $E_{\leq \leq k}(D) \geq$ $3\left(\begin{array}{c}k+3 \\ 3\end{array}\right)$ for all $0 \leq k \leq\left\lfloor\frac{n}{2}\right\rfloor-2$ with respect to $F$, then $\operatorname{cr}(D) \geq H(n)$.

If a vertex $v$ is incident to the reference face, the edges incident to $v$ have a predetermined distribution of $k$-values.

Lemma 2. 11 Let $D$ be a good drawing of $K_{n}, F \in \mathcal{F}(D)$ and $v \in V$ be a vertex incident to $F$. With respect to $F$, vertex $v$ is incident to two $i$-edges for $0 \leq i \leq\left\lfloor\frac{n}{2}\right\rfloor-2$. Furthermore, if we label the edges incident to $v$ counter clockwise with $e_{0}, \ldots, e_{n-2}$ such that $e_{0}$ and $e_{n-2}$ are incident to the face $F$, then $e_{i}$ is a $k$-edge with $k=\min (i, n-2-i)$ for $0 \leq i \leq n-2$.

Examples for lemma 2 are the vertices incident to $F$ in figure 1 . We denote the cumulated $k$-values for edges incident to a vertex $v$ in a drawing $D$ with $E_{\leq \leq k}(D, v)$. Due to lemma 2 it follows that $E_{\leq \leq k}(D, v)=\sum_{i=0}^{k}(k+1-i) \cdot 2=$ $2\left(\begin{array}{c}k+2 \\ 2\end{array}\right)$.

Next, we introduce invariant $k$-edges. Consider removing a vertex $v \in V$ from a good drawing $D$ of $K_{n}$, resulting in the subdrawing $D-v$. By deleting $v$ and its incident edges every remaining edge loses one triangle, i.e. for an edge $u w \in E$ there are only $(n-3)$ triangles $u w x$ with $x \in V \backslash\{u, v\}$ (instead of the $(n-2)$ triangles in drawing $D)$. The $k$-value of any edge $e \in E$ is defined as the minimum count of + or - oriented triangles that contain $e$. If the lost triangle had the same orientation as the minority of triangles, the $k$-value of $e$ is reduced by one else it stays the same. Therefore, every $k$-edge in $D$ with respect to $F \in \mathcal{F}(D)$ is either a $k$-edge or a $(k-1)$-edge in the subdrawing $D-v$ with respect to $F^{\prime} \in \mathcal{F}(D-v)$ and $F \subseteq F^{\prime}$. We call an edge $e$ invariant if $e$ has the same $k$-value with respect to $F$ in $D$ as for $F^{\prime}$ in $D^{\prime}$. We denote the number 
of cumulated invariant $k$-edges between $D$ and $D^{\prime}$ (with respect to $F$ and $F^{\prime}$ respectively) with $I_{\leq k}\left(D, D^{\prime}\right)$, i.e. $I_{\leq k}\left(D, D^{\prime}\right)$ equals the sum of the number of invariant $i$-edges for $0 \leq i \leq k$.
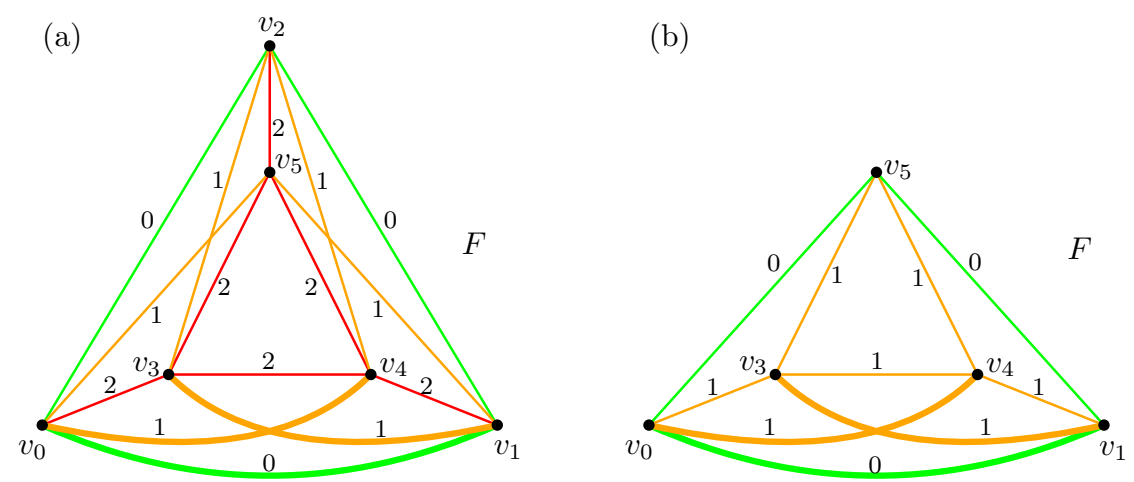

Fig. 1. Example: (a) shows a crossing optimal drawing $D$ of $K_{6}$ with the $k$-values at the edges. (b) shows the subdrawing $D-v_{2}$ and its $k$-values. The fat highlighted edges $v_{0} v_{1}, v_{0} v_{4}$ and $v_{1} v_{3}$ are invariant and keep their $k$-values. The reference face is the outer face $F$.

For a good drawing $D$ of $K_{n}$, we are able to express the value of cumulated $k$-edges with respect to a reference face $F \in \mathcal{F}(D)$ recursively by adding up the cumulated $(k-1)$-value of a subdrawing $D-v$, the contribution of the edges incident to $v$ and the number of invariant edges between $D$ and $D-v$.

Lemma 3. [5] Let $D$ be a good drawing of $K_{n}, v \in V$ and $F \in \mathcal{F}(D)$. With respect to the reference face $F$, we have

$$
E_{\leq \leq k}(D)=E_{\leq \leq k-1}(D-v)+E_{\leq \leq k}(D, v)+I_{\leq k}(D, D-v) .
$$

Ábrego et al. 5] use an inductive proof over $k$ to show that for a bishellable drawing $D$ of $K_{n} E_{\leq \leq k}(D) \geq 3\left(\begin{array}{c}k+3 \\ 3\end{array}\right)$ for all $k \in\left\{0, \ldots,\left\lfloor\frac{n}{2}\right\rfloor-2\right\}$. Together with lemma 1 follows $\operatorname{cr}(D) \geq H(n)$.

Here, we also use lemma 3 and show that for a seq-shellable drawing $D$ of $K_{n}$ the lower bounds on $E_{\leq \leq k}(D)$ hold for all $k \in\left\{0, \ldots,\left\lfloor\frac{n}{2}\right\rfloor-2\right\}$. But in contrast to [5], we use a more general and at the same time easy to follow approach to guarantee lower bounds on the number of invariant edges $I_{\leq k}(D, D-v)$ for $0 \leq k \leq\left\lfloor\frac{n}{2}\right\rfloor-2$.

\section{Seq-Shellability}

Before we proceed with the definition of seq-shellability, we introduce simple sequences. 


\subsection{Simple sequences}

We use simple sequences to guarantee a lower bound of the number of invariant edges in the recursive formulation of the cumulated $k$-value.

Definition 3 (Simple sequence). Let $D$ be a good drawing of $K_{n}, F \in \mathcal{F}(D)$ and $v \in V$ with $v$ incident to $F$. Furthermore, let $S_{v}=\left(u_{0}, \ldots, u_{k}\right)$ with $u_{i} \in$ $V \backslash\{v\}$ be a sequence of distinct vertices. If $u_{0}$ is incident to $F$ and vertex $u_{i}$ is incident to a face containing $F$ in subdrawing $D-\left\{u_{0}, \ldots, u_{i-1}\right\}$ for all $1 \leq i \leq k$, then we call $S_{v}$ simple sequence of $v$.

Before we continue with a result for lower bounds on the number of invariant edges using simple sequences, we need the following lemma.

Lemma 4. Let $D$ be a good drawing of $K_{n}, F \in \mathcal{F}(D)$ and $u, v \in V$ with $u$ and $v$ incident to $F$. The edge uv touches $F$ either over its full length or not at all (except its endpoints).

Proof. Assume that $D$ a is good drawing of $K_{n}$ in which the edge $u v$ touches $F$ only partly. We can exclude the case that an edge cuts a part out of $u v$ by crossing it more than once due to the goodness of the drawing (see figure 2 (a)). The case that an edge crosses the whole face $F$ and separates it into two faces is also impossible, because this would contradict that both $u$ and $v$ are incident to $F$. Therefore, a vertex $x$ has to be on the same side of $u v$ as $F$ and a vertex $y$ on the other side such that the edge $x y$ crosses $u v$. But the edge $x u$ cannot cross any edge $u z$ with $z \in V \backslash\{u\}$ as this would contradict the goodness of $D$ and $x u$ cannot leave the superface of $x$ without separating $v$ from $F$ (see figure 2(b) and (c)). We have the symmetric case for $v$. Consequently, $u v$ cannot touch $F$ beside its endpoints $u$ and $v$ (see figure 2 (d)), a contradiction to the assumption.

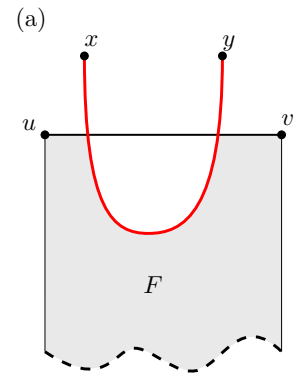

(b)

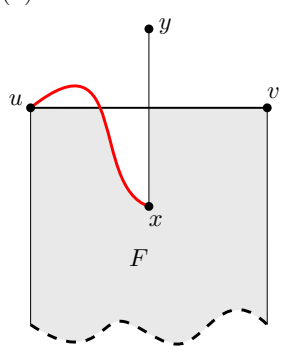

(c)

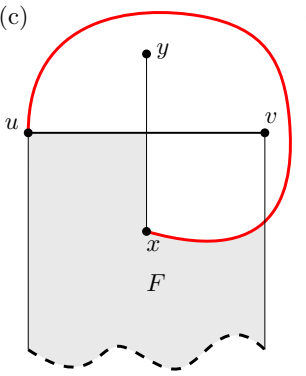

(d)

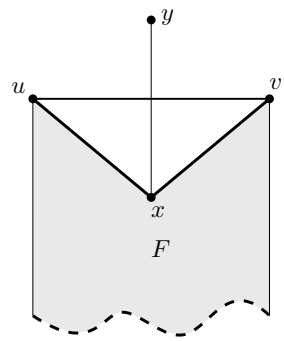

Fig. 2. (a) Due to the goodness of $D$ an edge cannot cut a part out of the edge $u v$. (b) The edges $u v$ and $u x$ cross, both have vertex $u$ as endpoint thus the drawing is not good. (c) The drawing is good but vertex $v$ is not incident to the face $F$. (d) The edge $u v$ is crossed, the drawing is good and both vertices $u$ and $v$ is are incident to $F$, however $u v$ is not incident to $F$. 
Corollary 1. Let $D$ be a good drawing of $K_{n}, F \in \mathcal{F}(D)$ and $u, v \in V$ with both $u$ and $v$ incident to $F$. If and only if $u v$ is a $j$-edge, there are exactly $j$ or $n-2-j$ vertices on the same side of uv as the reference face $F$.

The following lemma provides a lower bound for the number of invariant edges in the case that $F$ is incident to at least two vertices and we remove one of them.

Lemma 5. Let $D$ be a good drawing of $K_{n}, F \in \mathcal{F}(D)$ and $v, w \in V$ with $v$ and $w$ incident to $F$. If we remove $v$ from $D$, then $w$ is incident to at least $\left\lfloor\frac{n}{2}\right\rfloor-1$ invariant edges.

Proof. We label the edges incident to $w$ counter clockwise with $e_{0}, \ldots, e_{n-2}$ such that $e_{0}$ and $e_{n-2}$ are incident to the face $F$, and we label the vertex at the other end of $e_{i}$ with $u_{i}$. Furthermore, we orient all edges incident to $w$ as outgoing edges. Due to lemma 2 we know that $w$ has two $i$-edges for $0 \leq i \leq\left\lfloor\frac{n}{2}\right\rfloor-2$. Edge $e_{i}$ obtains its $i$-value from the minimum of say + oriented triangles and edge $e_{n-2-i}$ obtains its $i$-value from the minimum - oriented triangles (or vice versa). Assume that $v w$ is incident to $F$, i.e. $v w$ is a 0 -edge and all triangles $v w u$ for $u \in V \backslash\{v, w\}$ have the same orientation. Consequently, all $e_{i}$ or all $e_{n-2-i}$ for $0 \leq i \leq\left\lfloor\frac{n}{2}\right\rfloor-2$ are invariant. In the case that $v w$ is not incident to $F$ and is a $j$-edge, there are $j$ triangles $v w u_{h}$ with $u_{h} \in V \backslash\{v, w\}, 0 \leq h \leq j-1$ or $n-1-j \leq h \leq n-2$ and $u_{h}$ is on the same side of $v w$ as $F$ (corollary 1). This means, each triangle $w u_{h} v$ is part of the majority of orientations for the $k$-value of edge $w u_{h}$, therefore removing $v$ does not change the $k$-value and there are $j$ additional invariant edges incident to $w$ if we remove $v$.

The following lemma provides a lower bound for the number of cumulated invariant $k$-edges if we remove a vertex that has a simple sequence.

Lemma 6. Let $D$ be a good drawing of $K_{n}, F \in \mathcal{F}(D)$ and $v \in V$ with $v$ incident to $F$. If $v$ has a simple sequence $S_{v}=\left(u_{0}, \ldots, u_{k}\right)$, then

$$
I_{\leq k}(D, D-v) \geq\left(\begin{array}{c}
k+2 \\
2
\end{array}\right)
$$

with respect to $F$ and for all $k \in\left\{0, \ldots,\left\lfloor\frac{n}{2}\right\rfloor-2\right\}$.

Proof. Let $k \in\left\{0, \ldots,\left\lfloor\frac{n}{2}\right\rfloor-2\right\}$. We know that $u_{0}$ has at least $k+1 \leq\left\lfloor\frac{n}{2}\right\rfloor-1$ invariant edges with respect to $F$ and removing $v$. After removing vertex $u_{0}$ from drawing $D$, vertices $v$ and $u_{1}$ are incident to $F$. Since $k \leq\left\lfloor\frac{n}{2}\right\rfloor-2 \leq\left\lfloor\frac{n-1}{2}\right\rfloor-1$ and $u_{0}$ has an edge to $u_{1}$ in drawing $D$, vertex $u_{1}$ has at least $k$ invariant edges with respect to $F$ and removing $v$ in drawing $D$. In general, after removing vertices $u_{0}, \ldots, u_{i-1}$ from drawing $D$, vertices $v$ and $u_{i}$ are incident to $F$. For $u \in\left\{u_{0}, \ldots, u_{i-1}\right\}$ the edge $u u_{i}$ in drawing $D$ may be invariant or non-invariant, and we have $k+1-i \leq\left\lfloor\frac{n}{2}\right\rfloor-1-i \leq\left\lfloor\frac{n-i}{2}\right\rfloor-1$. Therefore, $u_{i}$ has at least $k-i+1$ invariant edges in drawing $D$ with respect to $F$ and removing $v$. Summing up 
leads to

$$
I_{\leq k}(D, D-v) \geq \sum_{i=0}^{k}(k+1-i)=\left(\begin{array}{c}
k+2 \\
2
\end{array}\right)
$$

\subsection{Seq-shellable drawings}

With help of simple sequences we define $k$-seq-shellability. For a sequence of distinct vertices $a_{0}, \ldots, a_{k}$ we assign to each vertex $a_{i}$ with $0 \leq i \leq k \leq n-2$ a simple sequence $S_{i}$, under the condition that $S_{i}$ does not contain any of the vertices $a_{0}, \ldots, a_{i-1}$.

Definition 4 (Seq-Shellability). Let $D$ be a good drawing of $K_{n}$. We call $D$ $k$-seq-shellable for $k \geq 0$ if there exists a face $F \in \mathcal{F}(D)$ and a sequence of distinct vertices $a_{0}, \ldots, a_{k}$ such that $a_{0}$ is incident to $F$ and

1. for each $i \in\{1, \ldots, k\}$, vertex $a_{i}$ is incident to the face containing $F$ in drawing $D-\left\{a_{0}, \ldots, a_{i-1}\right\}$ and

2. for each $i \in\{0, \ldots, k\}$, vertex $a_{i}$ has a simple sequence $S_{i}=\left(u_{0}, \ldots, u_{k-i}\right)$ with $u_{j} \in V \backslash\left\{a_{0}, \ldots, a_{i}\right\}$ for $0 \leq j \leq k-i$ in drawing $D-\left\{a_{0}, \ldots, a_{i-1}\right\}$.

Notice that if $D$ is $k$-seq-shellable for $k>0$, then the subdrawing $D-a_{0}$ is $(k-1)$ seq-shellable. Moreover, if $D$ is $k$-seq-shellable, then $D$ is also $j$-seq-shellable for $0 \leq j \leq k$.

Lemma 7. If $D$ is a good drawing of $K_{n}$ and $D$ is $k$-seq-shellable with $k \in$ $\left\{0, \ldots,\left\lfloor\frac{n}{2}\right\rfloor-2\right\}$, then $E_{\leq \leq k}(D) \geq 3\left(\begin{array}{c}k+3 \\ 3\end{array}\right)$.

Proof. We proceed with induction over $k$. For $k=0$ the reference face is incident to at least three 0-edges and it follows that

$$
E_{\leq \leq 0}(D) \geq 3=3\left(\begin{array}{c}
0+3 \\
3
\end{array}\right) .
$$

For the induction step, let $D$ be $k$-seq-shellable with $a_{0}, \ldots, a_{k}$ and the sequences $S_{0}, \ldots, S_{k}$. Consider the drawing $D-a_{0}$ which is $(k-1)$-seq-shellable for $a_{1}, \ldots, a_{k}$ and $S_{1}, \ldots, S_{k}$. Since $k-1 \leq\left(\left\lfloor\frac{n}{2}\right\rfloor-2\right)-1 \leq\left(\left\lfloor\frac{n-1}{2}\right\rfloor-2\right)$, we assume

$$
E_{\leq \leq k-1}\left(D-a_{0}\right) \geq 3\left(\begin{array}{c}
k+2 \\
3
\end{array}\right) .
$$

We use the recursive formulation introduced in lemma 3 , i.e.

$$
E_{\leq \leq k}(D)=E_{\leq \leq k-1}\left(D-a_{0}\right)+E_{\leq \leq k}\left(D, a_{0}\right)+I_{\leq k}\left(D, D-a_{0}\right) .
$$


Because $a_{0}$ is incident to $F$, we have $E_{\leq \leq k}\left(D, a_{0}\right)=2\left(\begin{array}{c}k+2 \\ 2\end{array}\right)$, and with the simple sequence $S_{0}$ of $a_{0}$ follows $I_{\leq k}\left(D, D-a_{0}\right) \geq\left(\begin{array}{c}k+2 \\ 2\end{array}\right)$ (see lemma 6). Together with the induction hypothesis, we have

$$
E_{\leq \leq k}(D) \geq 3\left(\begin{array}{c}
k+2 \\
3
\end{array}\right)+2\left(\begin{array}{c}
k+2 \\
2
\end{array}\right)+\left(\begin{array}{c}
k+2 \\
2
\end{array}\right)=3\left(\begin{array}{c}
k+3 \\
3
\end{array}\right) .
$$

Using lemmas 1 and 7 , we are able to verify the Harary-Hill conjecture for seqshellable drawings.

Theorem 1. If $D$ is a good drawing of $K_{n}$ and $D$ is $\left(\left\lfloor\frac{n}{2}\right\rfloor-2\right)$-seq-shellable, then $\operatorname{cr}(D) \geq H(n)$.

Proof. Let $D$ be a good drawing of $K_{n}$ and $\left(\left\lfloor\frac{n}{2}\right\rfloor-2\right)$-seq-shellable. Since $D$ is $\left(\left\lfloor\frac{n}{2}\right\rfloor-2\right)$-seq-shellable, it is also $k$-seq-shellable for $0 \leq k \leq\left\lfloor\frac{n}{2}\right\rfloor-2$. We apply lemma 7 and have $E_{\leq \leq k}(D) \geq 3\left(\begin{array}{c}k+3 \\ 3\end{array}\right)$ for $0 \leq k \leq\left\lfloor\frac{n}{2}\right\rfloor-2$ and the result follows with lemma 1.

If a drawing $D$ of $K_{n}$ is $\left(\left\lfloor\frac{n}{2}\right\rfloor-2\right)$-seq-shellable, we omit the $\left(\left\lfloor\frac{n}{2}\right\rfloor-2\right)$ part and say $D$ is seq-shellable. The class of seq-shellable drawings contains all drawings that are $\left(\left\lfloor\frac{n}{2}\right\rfloor-2\right)$-seq-shellable.

Theorem 2. The class of seq-shellable drawings strictly contains the class of bishellable drawings.

Proof. First, we show that $k$-bishellability implies $k$-seq-shellability. Let $D$ be a $k$-bishellable drawing of $K_{n}$ with the associated sequences $a_{0}, \ldots, a_{k}$ and $b_{0}, \ldots, b_{k}$. In order to show that $D$ is $k$-seq-shellable, we choose $a_{0}, \ldots, a_{k}$ as vertex sequence and $k$ simple sequences $S_{i}$ for $0 \leq i \leq k$ such that $S_{i}=$ $\left(b_{0}, \ldots, b_{k-i}\right)$. We assign simple sequence $S_{i}$ to vertex $a_{i}$ for each $0 \leq i \leq k$ and see that $D$ is indeed seq-shellable. Furthermore, drawing $H$ of $K_{11}$ in figure 3 is not bishellable but seq-shellable. It is impossible to find sequences $a_{0}, \ldots, a_{3}$ and $b_{0}, \ldots, b_{3}$ in $H$ that fulfill the definition of bishellability. However, $H$ is seq-shellable for face $F$, vertex sequence $\left(v_{0}, v_{2}, v_{3}, v_{4}\right)$ and the simple sequences $S_{0}=\left(v_{1}, v_{2}, v_{7}, v_{4}\right), S_{1}=\left(v_{1}, v_{8}, v_{6}\right), S_{2}=\left(v_{1}, v_{8}\right)$ and $S_{3}=\left(v_{1}\right)$.

The distinctive difference between seq-shellability and bishellability is that the latter demands a symmetric structure in the sense that we can mutually exchange the sequences $a_{0}, \ldots, a_{k}$ and $b_{0}, \ldots, b_{k}$. Thus, the sequence $b_{0}, \ldots, b_{k-i}$ has to be the simple sequence of $a_{i}$ in the subdrawing $D-\left\{a_{0}, \ldots, a_{i-1}\right\}$ for all $0 \leq i \leq k$ and vice versa, i.e. the sequence $a_{0}, \ldots, a_{k-i}$ has to be the simple sequence of $b_{i}$ in the subdrawing $D-\left\{b_{0}, \ldots, b_{i-1}\right\}$ for all $0 \leq i \leq k$. With seqshellability we do not have this requirement. Here we have the vertex sequence $a_{0}, \ldots, a_{k}$ and each vertex $a_{i}$ with $0 \leq i \leq k$ has its own (independent) simple sequence $S_{i}$.

Figure 5 shows a gadget that visualizes the difference between bishellability and seq-shellability: (a) shows a substructure with nine vertices that may occur 


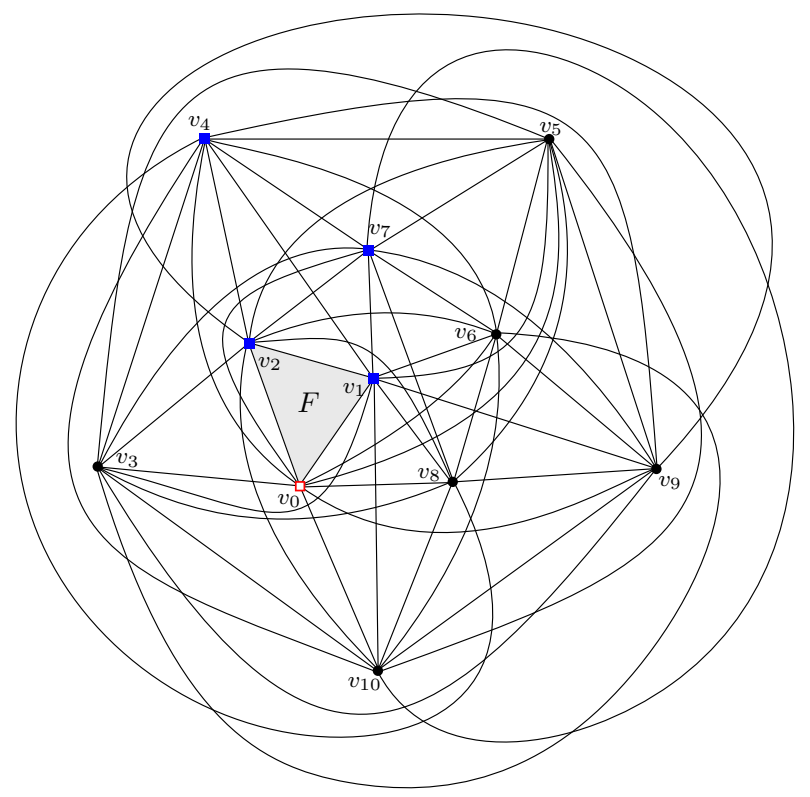

Fig. 3. Drawing $H$ of $K_{11}$ which is not bishellable for any face, however it is seqshellable for face $F$, vertex sequence $\left(v_{0}, v_{2}, v_{3}, v_{4}\right)$ and the simple sequences $S_{0}=$ $\left(v_{1}, v_{2}, v_{7}, v_{4}\right), S_{1}=\left(v_{1}, v_{8}, v_{6}\right), S_{2}=\left(v_{1}, v_{8}\right)$ and $S_{3}=\left(v_{1}\right)$. Vertex $v_{0}$ and the vertices of $S_{0}$ are highlighted as unfilled and filled squares.

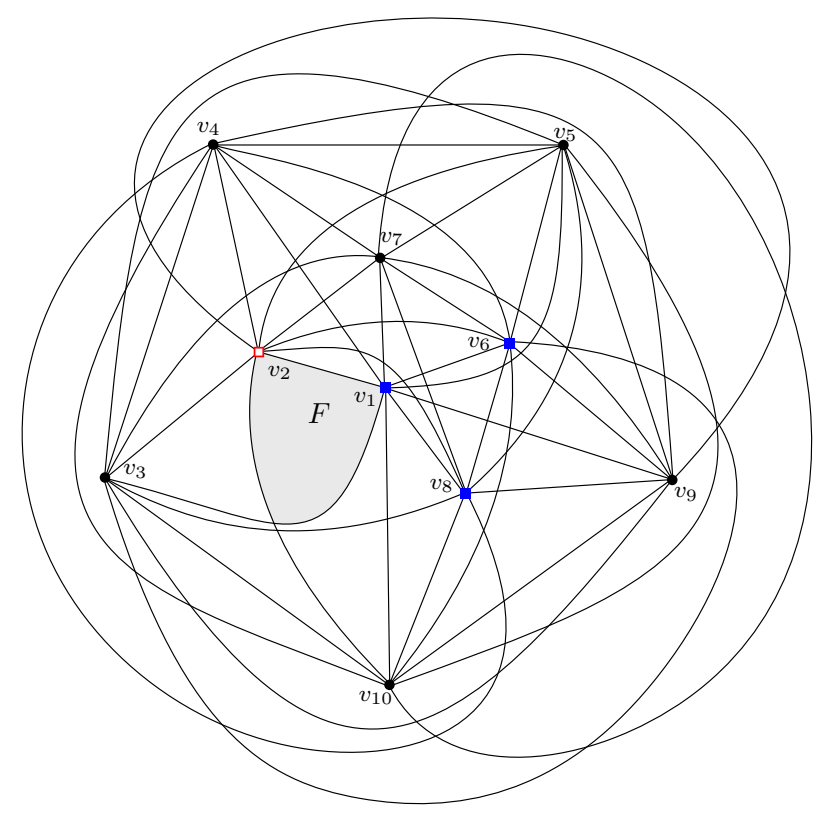

Fig. 4. Subdrawing $H-v_{0}$ after removing vertex $v_{0}$ and its incident edges. The second vertex of the vertex sequence $v_{2}$ is incident to the face containing $F$ and has simple sequence $S_{1}$. Vertex $v_{2}$ and the vertices $69 S_{1}$ are highlighted as unfilled and filled squares. 
in a drawing. We have the simple sequence $v_{1}, v_{2}, v_{4}$ for vertex $v_{3}$ in (b) and (c). Therefore, we can remove vertex $v_{3}$ and are able to guarantee the number of invariant edges. After removing vertex $v_{3}$ in (d), there are simple sequences for vertex $v_{1}$ and $v_{2}$, thus the substructure is seq-shellable. However, it is impossible to apply the definition of bishellability. We may use, for example, sequence $v_{1}, v_{2}, v_{4}$ as $a_{0}, \ldots, a_{k}$ sequence and we need a second sequence (the $b$ sequence) that satisfies the exclusion condition of the bishellability, i.e. for each $i \in\{0, \ldots, k\}$, the set $\left\{a_{0}, a_{1}, \ldots a_{i}\right\} \cap\left\{b_{k-i}, b_{k-i-1}, \ldots, b_{0}\right\}$ has to be empty (see definition 1). The first vertex of our second sequence (i.e. $b_{0}$ ) has to be $v_{3}$, because $b_{0}$ has to be incident to $F$. Now, for the second vertex we have to satisfy $\left\{a_{0}, a_{1}\right\} \cap\left\{b_{1}, b_{0}\right\}=\emptyset$, thus the second vertex has to be different from the first two vertices of the sequence $v_{1}, v_{2}, v_{4}$. Because we only can choose between vertices $v_{1}$ and $v_{2}$, we cannot select a second vertex for our $b$ sequence. Thus, the structure is not bishellable. We can argue the same way for the other possible sequences in the gadget.
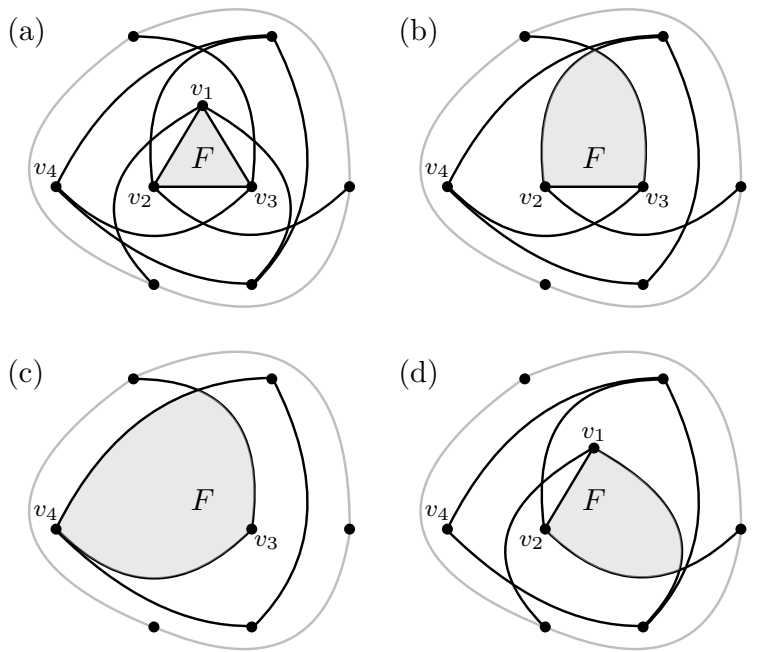

Fig. 5. The gadget does not allow for a bishellability sequence, because only one of the two sequences $a_{0}, \ldots, a_{k}$ or $b_{0}, \ldots, b_{k}$ can be chosen due to condition three of the definition of bishellability. However, the gadget is seq-shellable.

\section{Conclusion}

In this work, we introduced the new class of seq-shellable drawings and verified the Harary-Hill conjecture for this class. Seq-shellability is a generalization of bishellability, thus bishellability implies seq-shellability. In addition we exhibited a drawing of $K_{11}$ which is seq-shellable but not bishellable, hence seq-shellability 
is a proper extension of bishellability. So far, we are not aware of an optimal seq-shellable but non-bishellable drawing and we close with the following open questions:

1. Can we find a construction method to obtain optimal drawings of $K_{n}$ that are seq-shellable but not bishellable?

2. Does there exists a non-bishellable but seq-shellable drawing of $K_{n}$ with $10 \leq n<14$, such that after removing the first vertex of the simple sequence the drawing $D-a_{0}$ is still non-bishellable. We found a drawing of $K_{14}$ with this property.

\section{References}

1. Ábrego, B., Aichholzer, O., Fernández-Merchant, S., Ramos, P., Salazar, G.: The 2-page crossing number of $K_{n}$. In: Proceedings of the Twenty-eighth Annual Symposium on Computational Geometry. pp. 397-404. SoCG '12, ACM, New York, NY, USA (2012)

2. Ábrego, B.M., Aichholzer, O., Fernández-Merchant, S., Ramos, P., Salazar, G.: Shellable drawings and the cylindrical crossing number of $K_{n}$. Discrete \& Computational Geometry 52(4), 743-753 (2014)

3. Ábrego, B.M., Cetina, M., Fernández-Merchant, S., Leaños, J., Salazar, G.: On $\leq k$-edges, crossings, and halving lines of geometric drawings of $K_{n}$. Discrete \& Computational Geometry 48(1), 192-215 (2012)

4. Ábrego, B., Aichholzer, O., Fernández-Merchant, S., Hackl, T., Pammer, J., Pilz, A., Ramos, P., Salazar, G., Vogtenhuber, B.: All good drawings of small complete graphs. In: Proc. $31^{\text {st }}$ European Workshop on Computational Geometry (EuroCG). pp. $57-60(2015)$

5. Ábrego, B., Aichholzer, O., Fernández-Merchant, S., McQuillan, D., Mohar, B., Mutzel, P., Ramos, P., Richter, R., Vogtenhuber, B.: Bishellable drawings of $K_{n}$. In: Proc. XVII Encuentros de Geometría Computacional (EGC). pp. 17-20. Alicante, Spain (2017), https://arxiv.org/pdf/1510.00549.pdf

6. Balko, M., Fulek, R., Kyncl, J.: Crossing numbers and combinatorial characterization of monotone drawings of $K_{n}$. Discrete \& Computational Geometry 53(1), 107-143 (2015)

7. Erdös, P., Lovász, L., Simmons, A., Straus, E.G.: Dissection graphs of planar point sets. In: A Survey of Combinatorial Theory, pp. 139-149. Elsevier (1973)

8. Guy, R.K.: A combinatorial problem. Nabla, Bull. Malayan Math. Soc. 7, 68-72 (1960)

9. Harary, F., Hill, A.: On the number of crossings in a complete graph. Proceedings of the Edinburgh Mathematical Society 13(4), 333-338 (1963)

10. Lovász, L., Vesztergombi, K., Wagner, U., Welzl, E.: Convex quadrilaterals and k-sets. Contemporary Mathematics 342, 139-148 (2004)

11. McQuillan, D., Pan, S., Richter, R.B.: On the crossing number of $K_{13}$. J. Comb. Theory, Ser. B 115, 224-235 (2015)

12. Pan, S., Richter, R.B.: The crossing number of $K_{11}$ is 100 . Journal of Graph Theory 56(2), 128-134 (2007)

13. Schaefer, M.: The graph crossing number and its variants: A survey. The Electronic Journal of Combinatorics (Dec. 22, 2017) 1000, DS21-May (2013) 
14. Székely, L.A.: A successful concept for measuring non-planarity of graphs: The crossing number. Electronic Notes in Discrete Mathematics 5, 284-287 (2000)

15. Ábrego, B.M., Aichholzer, O., Fernández-Merchant, S., Ramos, P., Salazar, G.: More on the crossing number of $K_{n}$ : Monotone drawings. Electronic Notes in Discrete Mathematics 44, $411-414$ (2013) 